\section{Flexible Transistors with High Carrier Mobilities Made from Carbon Nanotubes}

Organic-based electronics are of great interest as a replacement for inorganic semiconductor devices, as the former are inexpensive, lightweight, and flexible, allowing the development of large flexible displays and electronic paper. However, progress in integrated organic-based electronic devices has been slow due to the low charge-carrier mobilities of these materials, which typically range from $\sim 0.1 \mathrm{~cm}^{2} / \mathrm{V} \mathrm{s}-$ for example, poly(3-hexylthiophene)to a maximum of $\sim 2 \mathrm{~cm}^{2} / \mathrm{V} \mathrm{s}$ for the best $p$-type crystalline organic semiconductors, with $n$-type carrier mobilities even lower. Carbon nanotubes show promise in overcoming these limitations because their carrier mobility exceeds even common semiconductor materials. Not only do carbon nanotubes exhibit very high strength, but their flexibility makes them a promising material for the development of large-scale flexible electronics. A team of researchers from Nanomix Inc. in Emeryville, Calif., recently demonstrated carbon nanotube-based $p$-type transistor networks supported on a flexible polymer substrate with an order of magnitude increase in hole-carrier mobility.

As reported in the October issue of Nano Letters, K. Bradley, J.-C.P. Gabriel, and G. Grüner of Nanomix manufactured and tested carbon nanotube network transistors on flexible polymer substrates. These polymer-supported networks show high durability during repeated bending and exhibit a hole-carrier mobility of $12 \mathrm{~cm}^{2} / \mathrm{V} \mathrm{s}$. The researchers used chemical vapor deposition to grow the carbon nanotube networks on 200-nm-thick silicon oxide layers on a Si substrate that consist of randomly oriented individual nanotubes rather than bundles. Using conventional lithography, the researchers patterned $\mathrm{Ti} / \mathrm{Au}$ source/drain contacts onto the nanotube networks (3.5 nm Ti followed by $50 \mathrm{~nm} \mathrm{Au} ; 200 \mu \mathrm{m}$ pads separated by $50 \mu \mathrm{m}$ gaps), resulting in a field-effect transistor network with on/off ratios as high as $10^{4}$. The scientists transferred the semiconducting networks to a flexible polymer substrate by spin-coating a $15 \mu \mathrm{m}$ polyimide film as the polymer support onto the silicon substrate, followed by a HF etch step to remove the $\mathrm{SiO}_{2}$ layer to induce device lift-off.

The devices were tested for their performance by placing the polyimide films on a metal chuck, which served as the gate electrode. The resulting device transfer characteristics exhibited large modulations in conductance for voltages of less than $100 \mathrm{~V}$, confirming that the polymer- supported networks behave as fieldeffect transistors. For a dielectric film as thick as $15 \mu \mathrm{m}$, these switching voltages are remarkably low, according to the researchers. The research team said that the low switching voltage is due to the high carrier mobility of the carbon nanotube network, with a measured hole mobility of $12 \mathrm{~cm}^{2} / \mathrm{V}$ s. This mobility is an order of magnitude improvement over the best currently used organic materials. The flexible network devices are remarkably resilient: A $60^{\circ}$ bending produces only a $12 \%$ decrease in conductance with full recovery over 12 bending cycles performed, despite the presence of multiple nanotube-nanotube junctions. The fabricated polymer-supported network transistors exhibit electronic properties that are similar to those of more conventional, silicon-supported carbon nanotube devices; they are flexible and could be made inexpensively.

Alfred A. ZinN

\section{Fluorescence from Individual Single-Walled Carbon Nanotubes Is Not Intermittent}

Potential photonic applications of singlewalled carbon nanotubes (SWNTs) in- clude nanometer-scaled integrated electroluminescent devices. Development of nanodevices, by their very nature, would benefit from the determination of specific optical properties of individual molecules, such as the true spectral linewidth. Until now, only ensembleaveraged spectra of SWNTs, in which spectral details are obscured, have been reported. However, a research team has reported fluorescence spectra from individual SWNTs. Their findings were published in the September 5 issue of Science.

University of Rochester and Universität Siegen researcher A. Hartschuh, together with a team of researchers, isolated individual nanotubes by spin-coating a SWNT suspension onto a glass coverslip. Atomic force microscopy showed that short SWNTs, with lengths of 200-300 nm, predominated. A density of about 10-20 Raman-active nanotubes per $100 \mu \mathrm{m}^{2}$ was determined with confocal Raman imaging. Laser excitation at $633 \mathrm{~nm}$ ensured that Raman signals (between $633 \mathrm{~nm}$ and 770 $\mathrm{nm}$ ) were isolated from the fluorescence signals above $850 \mathrm{~nm}$.

Using simultaneous resonance Raman and fluorescence measurements, the researchers found that individual nanotubes

\section{GLASSMAN HIGH VOLTAGE DC Power Supply Solutions for Vacuum Processing Applications \\ For small DC Sputtering sources, DC bias, Ion sources and Glow Discharge Plasma Requirements}

For over 25 years the Glassman name is synonymous with High Reliability Performance and has built it's reputation as a market leader by it's dedication and investment in technology development as well as after-sale customer support that is second to none in the power supply industry.

For Vacuum processing applications, Glassman's $\mathrm{FX}(300 \mathrm{~W}), \mathrm{EK}(600 \mathrm{~W})$ and $\mathrm{EQ}(1.2 \mathrm{~kW})$ series power supplies will meet your needs for low-mid power requirements, with typical application output voltage range from $300 \mathrm{~V}-10 \mathrm{kV}$. All feature compact size, advanced arc management, low stored energy, and use air as the primary dielectric-A Glassman Innovation! Key Features:

- Proprietary Arc Sense and Arc Quench circuitry for ultimate power supply and load protection

- High frequency technology-up to $60 \mathrm{kHz}$

- Tight regulation and high stability

- Low stored output energy

- RS-232 serial interface option

For more information on the complete line of Glassman High Voltage power supplies, visit our website at www.glassmanhv.com or call us today at $908-638-3800$.
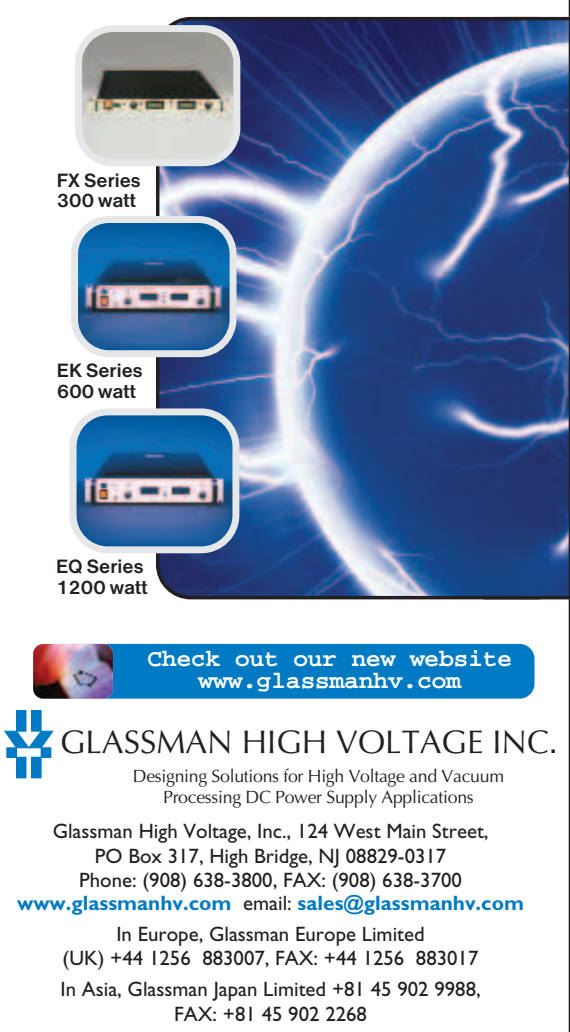

For more information, see http://advertisers.mrs.org 


\section{Know the Ups and Downs ...}

\section{Steep slope}

+ Big height difference

+ Large scan field

The LSM 5 PASCAL confocal laser scanning microscope broadens your microscopic horizons.

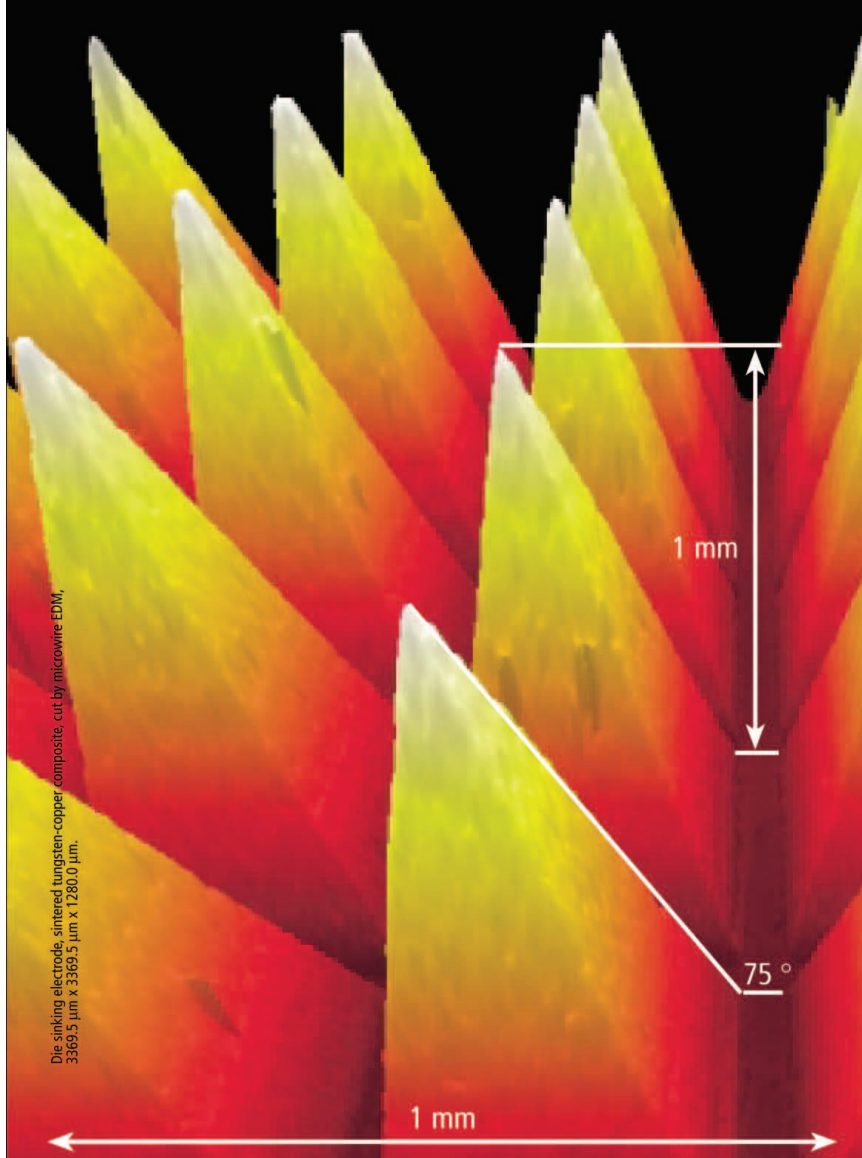

Highly resolved images. Simple and efficient imaging. Non-destructive.

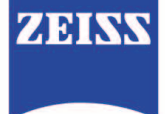

with identical structures - that is, identical diameters and chiralities-display different emission energies, although each individual emission line does match transitions observed in ensembleaveraged spectra. The researchers used low-energy Raman scattering attributed to the nanotube's radial breathing mode to verify that the source of the fluorescence was individual nanotubes. The researchers attribute the heterogeneous emission properties obtained from SWNTs with identical diameter and chirality values to localized defects and perturbations.

For moderate excitation energies $\left(<70 \mathrm{~kW} \mathrm{~cm}^{-2}\right)$, time traces of the fluorescence of individual SWNTs showed constant amplitude for $100 \mathrm{~s}$. The researchers said that by contrast both molecular dyes and individual semiconducting quantum dots exhibit fluorescence emission intermittencies or an on/off blinking behavior over very long time scales. The researchers conclude that "SWNTs have the potential to provide a stable, single-molecule infrared photon source with extremely narrow linewidth."

STEVEN TROHALAKI

\section{Three-Phase Boundary Fuel-Cell Reactor \\ Produces $\mathrm{H}_{2} \mathrm{O}_{2}$ at $93 \%$ Selectivity}

Industrial production of hydrogen peroxide $\left(\mathrm{H}_{2} \mathrm{O}_{2}\right)$ requires separation and distillation from a solution of alkylated anthraquinones (from crude oil) in a multistep procedure that consumes a lot of energy. For certain applications, a costly electrolysis process is used, but this option is not commonplace for high-scale production. Uses of $\mathrm{H}_{2} \mathrm{O}_{2}$ include water and wastewater treatment, bleaching of textiles and paper, food processing, and even odor control. To create a cost-effective and energy-efficient system suitable for large-scale production of $\mathrm{H}_{2} \mathrm{O}_{2}$, I. Yamanaka and his colleagues at the Tokyo Institute of Technology have designed a fuel-cell reactor with a three-phase boundary that overcomes the disadvantages of previous designs. With a three-phase boundary formed by gaseous $\mathrm{O}_{2}$, aqueous electrolyte, and a solid porous cathode, this reactor reduces the chance of $\mathrm{O}_{2}$ and $\mathrm{H}_{2}$ coming into contact and exploding; it generates electricity along with $\mathrm{H}_{2} \mathrm{O}_{2}$.

As reported in the August 8 issue of Angewandte Chemie, both the cathode and anode were fabricated from hot-pressed vaporgrown carbon fiber and poly(tetrafluoroethylene) powder, adding a small amount of a carbon black material to the cathode and platinum black powder to the anode. Pure $\mathrm{O}_{2}$ and $\mathrm{H}_{2}$ are the starting gases, and titration against $\mathrm{KMnO}_{4}$ determined the concentration of $\mathrm{H}_{2} \mathrm{O}_{2}$. A cation membrane separated the electrolyte compartment between the cathode and the anode into two sections, each one filled with $\mathrm{NaOH}$. Diffusion of $\mathrm{H}_{2} \mathrm{O}$ to the cathode compartment under stagnant conditions depleted the anode compartment and reduced the current density. Pumping $\mathrm{NaOH}$ continuously into the anode compartment helped stabilize the current density. Electrochemical measurements determined that the rate-controlling reaction was the reduction of $\mathrm{O}_{2}$. Using this reactor, $\mathrm{H}_{2} \mathrm{O}_{2}$ selectivity based on $\mathrm{H}_{2}$ or current efficiency based on the two-electron reaction is $93 \%$ after $2 \mathrm{~h}$, the rate of formation is $2.0 \mathrm{mmol} / \mathrm{h} \mathrm{cm}^{2}$, the concentration is $7.0 \mathrm{wt} \%$, and the current density is $100 \mathrm{~mA} / \mathrm{cm}^{2}$, similar to that obtained by electrolysis $\left(80-120 \mathrm{~mA} / \mathrm{cm}^{2}\right)$. By substituting $\mathrm{O}_{2}$ for air, the current efficiency is $88 \%$ after $3 \mathrm{~h}$, the rate of formation is $1.3 \mathrm{mmol} / \mathrm{h} \mathrm{cm}{ }^{2}$, the concentration is $6.5 \mathrm{wt} \%$, and the current density is $78 \mathrm{~mA} / \mathrm{cm}^{2}$, which is a slight decrease but still a good performance yet less expensive, representing an advantage for industrial-scale production.

SIARI SOSA

\section{Single-Molecule Resistance Measured by Repeated Formation of Molecular Junctions}

In the process of using individual molecules to create electronic circuits, the resistance level of a simple molecule like an alkane 